\title{
The Design about Reactive Compensation Complete Equipment of Combining Voltage Regulator with Magnetic Controllable Reactor
}

\author{
D.-L. Song, M.-M. Huang \\ Institute of Science, Information Engineering University, Zhengzhou, China (dongling120011@sina.com)
}

\begin{abstract}
This paper analyzes the existing reactive compensation control devices, then it proposes a new type of dynamic reactive power compensation scheme which is based on pressure regulating reactive compensation and magnetic controllable reactor. It Introduces principles and characteristics of the pressure regulating reactive compensation and magnetic controllable reactor, then expounds control principle of combined dynamic reactive power compensation device and its running effect in power system . The combined dynamic reactive power compensation device conforms to the development direction of power system, and has good prospects for development.
\end{abstract}

Keywords - pressure regulating reactive compensation, dynamic reactive power compensation, MCR, voltage stability, electrical power system

\section{调压与磁控电抗器组合式无功补偿成套装置的设计}

\author{
宋冬灵黄丞沝 \\ 信息工程大学理学院, 郑州, 河南, 中国
}

摘 要 通过分析现有无功补偿控制装置的不足, 提出了基于调压式无功补偿和磁控电抗器的新型动态无功补 偿方案。介绍了调压式无功补偿、磁控电抗器的原理及特性, 阐述了基于调压与磁控电抗器相结合的组合式动态无 功补偿装置的控制原理及其在电力系统中的运行效果。组合式无功补偿装置符合电力系统的发展方向, 具有良好的 发展前景。

关键词 调压式无功补偿, 动态无功补偿, 磁控电抗器 (MCR), 电压稳定, 电力系统

\section{1. 引言}

随着我国国民经济的不断发展, 各行各业对电能质 量的需求量与日剧增, 电力负荷越来越大。由于感性负 荷较大，造成电网功率因数降低，损耗增大，能源利用 率降低，供电质量下降。因此，搞好无功补偿，提高功 率因数, 降低供电损耗, 提高供电质量, 是解决问题的 重点。

目前我国电力系统所采用的无功补偿主要以固定电 容器补偿方式为主。电容器无功补偿主要有单组或多组 投切固定补偿方式、可控电抗器配合调节等方式。这些 方式中, 电容器均是全电压投切, 或是少数调压式无功 补偿方式。较大容量无功补偿装置一般采用分组投切方 式, 这种方式在分闸时易产生操作过电压, 合闸时易产
生较大涌流, 对电网、电容器和断路器冲击很大, 极易 造成电容器、开关的损伤; 其电气寿命、机械寿命都大 大降低。安装补偿容量的确定也是一个难题, 如果选取 的容量过大, 负荷不大, 整组投入过补, 电压偏高; 不 投时功率因数偏低; 如果容量较小, 则补偿容量不足。 传统无功补偿装置无自控装置, 人员不能全面照顾变化 情况, 电容器投切不及时, 易造成电压、功率因数指标 不合格。

调压式动态无功补偿装置能够较好的解决传统无功 补偿装置的问题, 但是调压过程是离散台阶变化的, 不 能实现真正的柔性补偿, 并且在最低负荷时可能会向电 网系统倒送容性无功功率。磁控电抗器 (MCR) 无功补偿 装置虽然补偿过程中是柔性补偿, 但是在最低负荷时, 
大容量的磁控电抗器需要发出大量的感性无功来抵消固 定电容器组发出的容性无功功率, 这样的运行方式严重 浪费能源。设计一套将调压与磁控电抗器结合的组合式 无功补偿装置, 可以使他们扬长避短, 达到更好的无功 补偿效果。

\section{2. 调压式无功补偿和磁控电抗器无功补偿}

\section{1 调压式无功补偿}

调压式无功补偿装置通过改变补偿电容器的电压来 改变补偿电容器发出的无功量。其补偿系统采用特殊设 计的深度调压变压器, 可实现大范围动态调压; 由微机 控制器构成的高压无功补偿装置, 通过实时采集电网的 电压、电流、功率因数, 分析负荷的变化趋势、系统无 功功率、系统谐波含量、电压波动情况等, 利用模糊控 制技术调节有载分接开关, 实现动态优化补偿, 并达到 无功补偿容量随系统负荷无功容量的变化自动跟踪的目 的, 从而实现了全自动跟踪动态无功补偿。并且与 SVC 相比, 该装置无谐波污染, 不需要辅加谐波吸收回路。 但是这种装置也有明显的不足, 它的调档过程还是离散 的, 即不能及时连续可调, 因此对于快速波动负荷不能 实时跟踪, 使得无功补偿效果不明显。在低负荷情况下, 该装置在最低档位时, 会向电网系统倒送容性无功, 使 得设备利用下降，并占用线路的有效资源。

\section{2 磁控电抗器无功补偿}

磁控电抗器的结构如图 1 所示, 磁控电抗器本体采 用三相一体化结构, 每相有 2 个工作铁心, 铁心具有截 面积较小的一段, 每个工作铁心上绕有交流主绕组和直 流控制绕组, 同相 2 个铁心柱上的交流主绕组并联后接 至电网, 三相绕组星形连接。三相控制绕组接成双三角 形, 在三角形顶点引出直流控制端; 主铁心都为磁阀式 结构。采用极限磁饱和技术, 在整个设计容量范围内, 大截面铁心段始终不饱和, 仅小截面段饱和, 通过改变 小截面段的磁路饱和程度来改变电抗器的容量, 这样大 大减少了电抗器谐波和有功损耗。整流器输出的直流控 制电流对铁心分别起到增磁和去磁作用, 以调节铁心磁 饱和度, 从而可以通过控制磁控电抗器的输出电流而达 到平滑调节电抗器容量的目的。

磁控电抗器采用可控硅控制, 其容量可以连续无级 调节, 消除了仅有电容器投切时的阶梯式无功补偿, 实 现无功的真正就地平衡, 降低网损, 提高系统的传输能 力。双向的无功功率补偿扩大了变电站无功调度的工作
范围, 达到了无功优化调节的目的, 为电网的无功控制 提供了有效手段。扩大了变电站的无功调节容量, 具有 更优越的电压调节效果, 减少了分接头的调整频次。

磁控电抗器在跟踪效果上比调压式高压动态无功补 偿要好, 响应速度在 $0.2 \mathrm{~S}$ 左右, 但是传统多采用固定电 容或电容分成两组进行补偿, 这样就会出现相应配置磁 控电抗器的容量增大, 并且不能减小投入时对电网和电 容器的冲击, 在负荷低谷时, 就会出现磁控电抗器的感 性无功发出较大, 来抵消电容器组发出的感性无功, 从 而浪费能源。
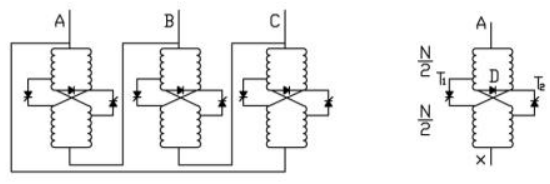

图 1 磁控电抗器结构图

\section{3. 组合式无功补偿装置}

为了弥补现有无功补偿装置的不足, 设计了一套新 型的调压式与磁控电抗器相结合的无功补偿装置 (下面 简称组合式无功补偿装置)。

\section{1 组合式无功补偿装置结构}

组合式无功补偿装置主要有两部分组成: 调压式无 功补偿部分 (有载自藕调压器) 和磁控电抗器部分, 其 结构如图 2 所示, 一次系统如图 3 所示。图中左部分是 调压无功补偿部分, 1 代表隔离开关、2 代表真空断路器、 3 代表调压器、4 代表串联电抗器、5 代表避雷器、6 代 表放电线圈、 7 代表电容器组。右部分是磁控电抗器, 1 代表隔离开关、8 代表磁控电抗器。

\section{2 组合式无功补偿装置工作原理}

组合式无功补偿装置可以直接接入 6-35KV 电压等 级的母线上, 变电站低压侧母线 (6-35KV) 通过电容器 出线开关柜的输入端连接, 调压器将母线与电容器组连 接起来, 在保证母线电压稳定的前提下, 改变电容器组 输出端电压, 从而保证电容器组输出容量满足系统要求。 磁控电抗器与调压器侧并联, 用于吸收电容器组或电力 系统多余的无功功率, 确保电力系统的无功功率平衡。 通过电容器出线开关柜分合无功补偿设备, 并且在无功 补偿设备有故障时及时切断故障点。隔离开关在检修设 备时可以看到明显的断点, 也可以把设备退出运行。调 压器起到调节电容器的端电压, 改变电容器组的无功容 
量的作用。串联电抗器可以抑制谐波合闸涌流。电容器 组是容性无功源。放电线圈保护电容器组, 当电容器组 退出运行时释放电荷。避雷器用于释放雷电以及过电压。 微机控制器通过采集图 3 中主变低压侧电压互感器和电 流互感器二次信号, 以及主变低压侧开关量信号、电容 器出线开关柜的开关量信号, 进行分析计算及判断, 发 出命令, 从而调

节变电站主变分接头, 调整低压侧母线电压, 保证 母线电压合格率。调节调压器的输出电压可以改变电容 器无功输出量, 调节磁控电抗器的导通角可以改变磁控 电抗器的感性无功输出量, 并具有相应的显示和信号功 能。

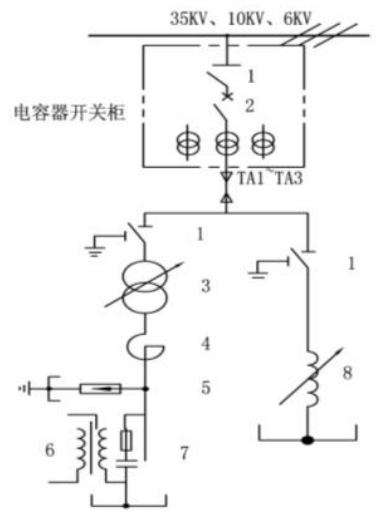

图 2: 组合式无功补偿装置结构图

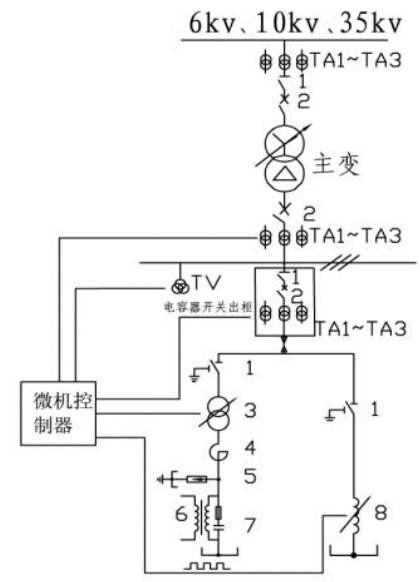

图 3: 组合式无功补偿装置一次系统图

通过微机控制器对信号的分析计算, 当系统功率因数低 于目标功率因数时, 微机控制器输出导通角增大, 磁控 输出的感性无功接近于零, 如果此时系统功率因数依然 低于目标功率因数, 微机控制器发出调档脉冲, 使得调 压器档位从低档位调到高档位, 功率因数合格。当系统 功率因数高于目标功率因数时, 程序计算出的过剩无功
与磁控电抗器的感性无功总量比较, 如果过剩无功大于 磁控电抗器的总容量, 微机控制器发出调压器控制器降 档脉冲, 使得调压器的档位从高档位向低档位调节, 如 果过剩无功小于磁控电抗器的总容量, 微机控制器输出 导通角减小, 磁控输出的感性无功增大, 直到过剩无功 与感性无功相等。

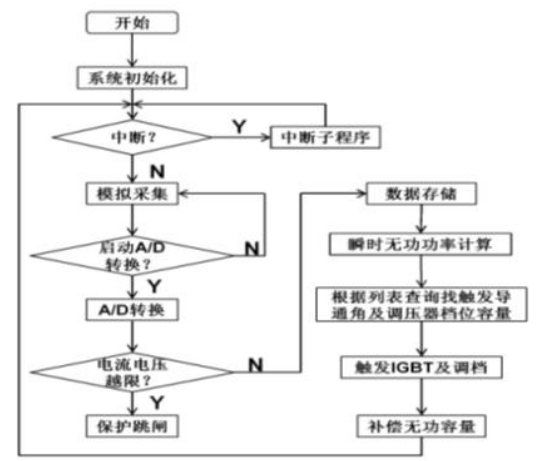

图 4: 组合式无功补偿装置的程序流程图

当主变低压侧母线电压低于目标电压时，微机控制 器发出调主变升档位脉冲, 使得主变档位从低档位调到 高档位，使得主变低压侧母线合格。当主变低压侧母线 电压高于目标电压时, 微机控制器发出调主变降档位脉 冲, 使得主变档位从高档位调到低档位, 使得主变低压 侧母线合格。

\section{3 组合式无功补偿装置结构}

组合式无功补偿装置具有以下特点:

(1) 采用有载自耦调压器调压与磁控电抗器组合, 调节速度快, 可实现实时互补自动调节, 补偿效果显著;

（2）可低电压合闸，大大减少了合闸涌流，有效 降低了对系统及电容器的冲击;

（3）装置不产生谐波，不会对系统造成谐波污染;

(4) 有串联电抗器时, 可保证每个档位的电抗率 恒定不变;

（5）采用小容量磁控电抗器有效降低大容量感性 无功在电网上的能源浪费, 并且节省磁控电抗器制造成 本，有效地增强了企业市场竞争力。

（6）有效降低了有载自耦调压器的动作次数。

(7) 附加损耗小, 仅为电容器容量的 $2 \%$ o左右, 相 当于 SVC 损耗的十分之一;

（8）装置自动化程度高，具有完善的保护功能、 数字通讯和远程维护功能, 可满足无人值班及免维护的 需求; 
（9）与投切式无功补偿装置相比, 仅一组电容器 组固定接入即可实现九档输出, 补偿精度高, 可满足系 统无功功率变化要求;

（10）与投切相比, 可保证电容器长期在额定电压 以下运行, 无投切过电压和涌流问题, 大大地延长了电 容器的使用寿命;

(11) 电容器无需分组投切, 减少了投切开关等设 备及占地面积，节约了基建投资成本。

组合式无功补偿装置有效结合了调压式多档位的 调节优点, 使得适合不同时刻负荷变化的需求, 与小容 量磁控电抗器快速响应真正柔性连续可调相结合, 弥补 了有载自耦调压器分接开关频繁动作以及低负荷时磁 控电抗器功耗的能源浪费。使得设备相互扬长避短, 更 加对电网系统的变化响应自如。

组合式无功补偿装置有效解决了调压式无功补偿 装置在调档过程中档位与档位的电容器无功功率的突 变补偿效果离散状态, 解决了调压式无功补偿装置在轻 载时最低档位时还向系统倒送无功情况, 解决了调压式 无功补偿装置不能适应负荷反复突变和连续快速变化 环境。更最要的是, 组合式无功补偿装置采用小容量磁 控电抗器, 在不影响快速响应的前提下, 有效地降低了 大容量磁控电抗器在电网运行状态下大容量感性无功 在电网上的能量浪费。

组合式无功补偿装置的程序流程如图 4 所示。

\section{4 案例分析}

该组合式装置对无功负荷较大、负荷变化较频繁、 电压低的变电站更为适应。使用前功率因数一般在 0.85 左右, 电压波动也较大, 变电站值班人员手动调节十分 麻烦, 且达不到满意的效果; 若使用该组合式装置, 预 计功率因数均可保持在 0.98 以上, 且电压保持在合格的 范围之内。

以变电站安装该组合式装置为例。

由于电容器断路器正常情况下不再频繁投切 (即使 投切也都在较小电流下), 因此, 可大大延长断路器的 检修周期。减少了断路器的维护费用, 增加了断路器使 用寿命。

由于有效降低了调压器的有载分接开关动作次数, 使得有载分接开关使用寿命延长 1.5 倍, 由于磁控电抗 器是电子开关, 维护成本低。

与常规补偿方式相比, 若变电站一段母线带 4 组电 容器, 使用组合式装置后, 可节省三组 $6 \mathrm{kV}$ （或 $10 \mathrm{kV}$ 、
$35 \mathrm{kV}$ ) 电容器出线柜 (包括开关、开关柜、隔离开关、 电流互感器、保护等设备及相应的一、二次电缆), 节 省费用在 24 万元以上 (仅估算节省装置的费用, 未考 虑节省的房屋设施费用)。

组合式装置能够跟踪变电站电网的运行情况, 实时 补偿无功功率, 使变电站保持较高功率因数 (预计可达 0.98 以上), 并且不会造成过补或欠补, 因此可以降低 网损, 提高供电能力和电能质量。若变电站主变为 $10000 \mathrm{kVA}$, 平均提高功率因数为 0.05 , 年损耗将能降低 $130 \mathrm{MWh}$; 电费按 0.3 元 $/ \mathrm{kWh}$ 计算, 企业可增收约 3.9 万元。

由于提高了安全运行可靠性, 减少了电容器故障及 由于电容器故障引起的电网故障, 提高了供电量。同 时, 由于提高了功率因数, 降低了无功电流, 可以增加 变电站负载能力。如增加有功负载 $110 \mathrm{~kW}$, 年增加供 电量 $964 \mathrm{MWh}$; 利润按 0.1 元 $/ \mathrm{kWh}$ 人民币计算, 企业可 增收约 9.64 万元。

通过以上几项计算, 使用组合式装置每年可为变电 站创造经济效益约 37 万元。

\section{4. 结论}

组合式无功补偿装置吸取了调压式和磁控电抗器 两种无功补偿装置的优点, 同时避免了他们各自的缺 点。该装置可以适合不同时刻变电站电网负荷变化的需 求, 可实现对无功补偿的柔性连续调节, 并且减少了能 源浪费。该套装置投运后对稳定系统电压和平衡无功发 挥将发挥重要作用, 具有良好的补偿效果。

组合式补偿是电能质量新一代理想产品, 有效地节约了 能源, 减少了浪费, 提高了设备利用率, 降低了设备重 复建设性。

\section{参考文献(References)}

[1] B.A.Wang, L.L.Jin, Y.Q.Luo, et al., "Dynamic reactive power compensation equipment based on magnetically controlled reactor," Electric Power Automation Equipment, vol. 35, no. 4, pp. 97-100, 2010.

[2] C.H. Li, "Application of magnetic contaolled reactor in the reactive power compensation of substation," Distribution \& Utilization, vol. 25, no. 3, pp. 41-43, 2008.

[3] D.J.Zhu, L.X.Huang, B.Huang, et al., "Automatic Regulation Equipment of The New-Type Voltage Adjustable Reactive Power," Power Capacitor, vol. 28, no.3, pp. 9-13, 2007.

[4] Y.B. Wei, T.Li, G.L. Zhang, et al., "A control method for the magnetic-valve controllable reactor based on instantaneous reactive power theory," Power System protection and Control, vol. 39, no.22, pp. 117-121, 2011. 